\title{
Isocinetisme et sport de haut niveau : Applications à la traumatologie du sport
}

\author{
Yannick Barthélémy ${ }^{1}$, Jean-François Kaux ${ }^{2}$ et Jean Marcel Ferret ${ }^{3}$ \\ 1 Centre de médecine du sport Charleroi Sport Santé, rue de Goutroux, 396031 Monceau sur Sambre, Belgique \\ 2 Service de Médecine de l'Appareil Locomoteur et de Traumatologie du sport, SPORTS² (Service Pluridisciplinaire Orthopédie \\ Rééducation Traumatologie Santé Sportif), CHU et université de Liège, avenue de l'hôpital, B35, 4000 Liège, Belgique \\ 3 SPOREA, Lyon, France
}

Reçu le 24 mai 2013 - Accepté le 6 septembre 2013

Résumé. Le sport de haut niveau par la pratique régulière de l'activité, la répétition de gestes stéréotypés à haute intensité et la spécificité de l'entraînement, expose les sportifs au risque de traumatismes. Risque qui a considérablement augmenté avec les exigences du haut niveau, la fréquence de plus en plus grande des compétitions, la pression du résultat et l'émergence du sport business. Face à ce risque, l'isocinétisme s'inscrit comme une technique de référence dans l'évaluation des performances musculaires. Le dynamomètre isocinétique permet une mesure précise et reproductible de la force musculaire dynamique, à condition de respecter une méthodologie rigoureuse. L'utilisation de l'isocinétisme dans le sport de haut niveau s'est nettement développée afin d'identifier les profils musculaires à risque, et corriger les déséquilibres musculaires, en prévention de blessures. En thérapeutique, nous utilisons l'isocinétisme pour la rééducation des pathologies musculaires, tendineuses et ligamentaires (rupture du LCAE). Aujourd'hui, le travail musculaire excentrique doit s'imposer dans les pathologies citées ci-dessus. Les modalités d'application du protocole conditionnent son efficacité. Nous prônons, depuis des années, une prise en charge précoce de la blessure par un travail isocinétique excentrique à vitesse lente, dans un but cicatriciel du tissu lésé. L'outil isocinétique permet de réaliser ce travail excentrique précocement de manière sécurisé. Il est complémentaire aux autres techniques kinésithérapiques. Il nous semble indispensable dans la prise en charge des sportifs de haut niveau pour le suivi de la rééducation et la complète récupération fonctionnelle. Il quantifie l'un des critères de reprise du sport permettant de réduire le risque de récidive tant redouté. Après chirurgie du ligament croisé antérieur, objectiver précisément le niveau de récupération musculaire permet de mieux individualiser la rééducation et la phase de réathlétisation.

Mots clés : Sport de haut niveau, isocinétisme, muscle, tendon, LCAE, travail excentrique

\section{Abstract. Isohinetic and high performance sport: application sport traumatology.}

The high performance sport through regular activity, repetition of stereotypical high intensity movements and specificity of training, outlines the risk of sports injuries. Risk has increased substantially with the requirements of the highest level, the frequency of growing competition, the pressure of the result and the emergence of the sports business. Given this risk, the isokinetic registers as a reference technique in the assessment of muscle performance. The isokinetic dynamometer allows accurate and reproducible measurement of dynamic muscle strength, provided you follow a rigorous methodology. The use of isokinetic in top-level sport has grown dramatically to identify risk profiles muscle, and correct muscle imbalances, prevent injury. In treatment we use for isokinetic rehabilitation of muscle, tendon and ligament (ACL tear) diseases. Today, the eccentric muscle work must prevail in the pathologies mentioned above. The implementing rules of the protocol determine its effectiveness. We advocate for years, an early treatment of the injury by eccentric isokinetic work at slow speed in a scar purpose of injured tissue. The isokinetic tool to achieve this eccentric work early so secure. It is complementary to other physiotherapy techniques. It seems essential in the management of high-level athletes to monitor rehabilitation and full functional recovery. It quantifies one of the criteria for return to play to reduce the risk of recurrence dreaded. After anterior cruciate ligament surgery, objectify precisely the level of muscle recovery to better individualize therapy and phase réathlétisation.

Key words: High performance sport, isokinetics, muscle, tendon, ACL, eccentric work 
Le sportif de haut niveau est un professionnel devant faire face à une charge de travail lourde et contraignante, avec parfois des souffrances physiques importantes. L'exigence de production de performances physiques implique un investissement total quotidien et à long terme. Il est immergé dans un processus mental dont les valeurs dominantes sont la compétition et l'élitisme. De par son statut de sportif de haut niveau, il présente des phénomènes psychoaffectifs spécifiques face à la blessure qui ont des conséquences quant à ces exigences de reprise d'activité aussi bien d'un point de vue chronologique que qualitatif.

De par nos expériences professionnelles, nous évoquerons ce que l'isocinétisme peut apporter dans la prise en charge des différentes pathologies musculaires, tendineuses, et ligamentaires (Croisier \& Crielaard, 2004). Le concept isocinétique propose un mouvement analytique unidirectionnel exécuté à une vitesse angulaire constante choisie par l'expérimentateur. Cette dernière caractéristique résulte de l'intervention d'une résistance variable, gérée par le dynamomètre et asservie en permanence aux capacités d'effort du sujet. L'évaluation simultanée des groupes agonistes et antagonistes (fléchisseurs et extenseurs du genou par exemple) présente un intérêt évident pour la détection des déséquilibres musculaires (Croisier \& Crielaard, 1999b). L'information isocinétique propose des courbes et paramètres chiffrés (Croisier \& Crielaard, 1999a, 1999b). L'analyse globale de la courbe renseigne sur la qualité de la contraction musculaire pour les différentes positions articulaires successives. Elle correspond, in vivo, à l'étude de la relation tension longueur musculaire et peut déceler d'éventuelles anomalies n'entraînant pas une modification systématique des paramètres chiffrés. Parmi les paramètres chiffrés proposés, citons :

- le moment de force maximum (MFM en N.m);

- le travail (W en $\mathrm{J})$;

- la puissance $(\mathrm{P}$ en $\mathrm{W})$;

- l'angle d'efficacité maximale du pic de couple de force (AEM en degrés).

Le bilan isocinétique s'interprète classiquement par l'analyse :

- du rapport agoniste/antagoniste, calculé sur la base de leur MFM respectif (exemple pour le genou $\mathrm{Fl} / \mathrm{Ex})$. Un ratio mixte $\mathrm{Fl}_{\text {excentrique/Ex concentrique }}$ $\left(\mathrm{Fl}_{\text {exc }} / \mathrm{Ex}_{\mathrm{conc}}\right)$, plus proche de la réalité fonctionnelle, complète les rapports classiques utilisant le même mode concentrique de contraction. Le ratio mixte est une combinaison du mode concentrique pour un groupe musculaire moteur et du mode excentrique pour l'autre groupe à vocation frénatrice. Plusieurs études proposent des ratios mixtes, dont celui développé par Croisier $\left(\mathrm{Fl}_{\text {exc } 30 \% \mathrm{~s}} / \mathrm{Ex}_{\text {conc } 240 \% \mathrm{~s}}\right)$ qui semble fort discriminant par rapport aux ratios concentriques dans la pathologie ischio-jambière (Croisier \& Crielaard, 1996). La valeur normale de ce ratio mixte est proche de 1 .
- les paramètres exprimés en valeurs relatives (MFM par unité de masse corporelle), comparés avec les valeurs normales d'une population de référence permettent de déterminer « une qualité » de force du sportif étudié qui varie en fonction du sport pratiqué et de la masse musculaire (Jidovtseff, Croisier, Mordant, \& Crielaard, 2005).

Parallèlement à l'évaluation, le dynamomètre isocinétique permet le renforcement musculaire dans divers contextes amyotrophiques tels que les lésions musculotendineuses et ligamentaires. L'adaptation permanente de la résistance, autorisant le développement d'un moment de force maximum sur l'amplitude complète du mouvement, rend la technique plus efficace que la seule méthode isotonique. L'asservissement de la charge imposée garantit des conditions de sécurité optimales. Cette qualité reste primordiale, particulièrement pour le mode excentrique où la machine impose le mouvement articulaire et force ainsi le muscle à l'allongement malgré sa contraction. Grâce à un mécanisme complémentaire, le dispositif isocinétique s'arrête immédiatement, quelle que soit la position, lorsque le patient interrompt la contraction. Le renforcement isocinétique apparaît hautement spécifique par l'individualisation des protocoles établis sur la base des anomalies objectivées lors de l'évaluation.

\section{Pathologie musculaire}

La pathologie musculaire figure parmi les causes majeures éloignant le sportif de son activité. L'UEFA a réalisé une étude sur 23 des 50 meilleures équipes européennes durant sept saisons de 2001 à 2008 (Ekstrand, Hägglund, \& Walden, 2011). L'incidence des blessures est de 27,5 lésions pour 1000 heures de match et 4,1 lésions pour 1000 heures d'entraînement. Il y a environ deux blessures par joueur durant la saison et la première déchirure d'ischio-jambier représente $17 \%$ des lésions. Il y a environ $12 \%$ de récidives dont l'arrêt est plus long (18 jours) que la lésion initiale.

Ekstrand et al. (2011) ont réalisé dernièrement une étude épidémiologique sur des équipes UEFA de 2001 à 2009. Les lésions musculaires représentent $31 \%$ de toutes les lésions et $27 \%$ des absences sportives. Quatrevingt douze pour-cent des lésions musculaires impliquent les quatre groupes musculaires du membre inférieur : IJ (37 \%), adducteurs (23\%), quadriceps (19\%) et triceps sural (13\%). Seize pour-cent des lésions musculaires sont des rechutes entraînantes des absences plus longues de $30 \%$. De ce fait, l'incapacité de pratique sportive est à l'origine de coûts et de pertes financières non négligeables : primes d'assurances accident et perte de gain plus élevées, revenus des clubs diminués par une attractivité moindre liée aux blessures de joueurs vedettes, diminution du nombre de spectateurs, des contrats de sponsoring ou de droits télévisés (Woods et al., 2002). Un programme de réhabilitation raisonnée des blessures musculaires est impératif afin 


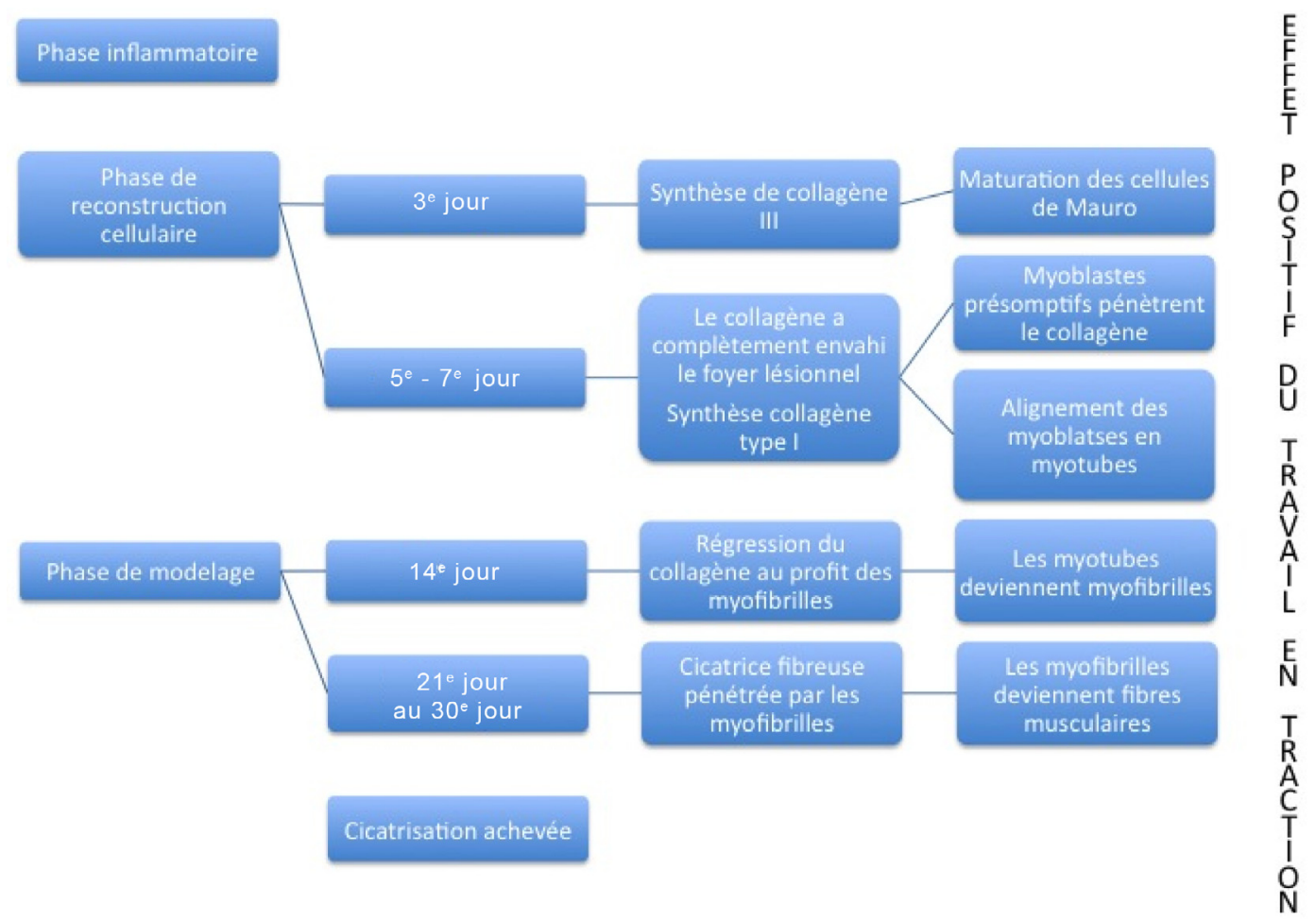

Fig. 1. D'après Processus de réparation en trois phase. Ferret JM (2010), $10^{\mathrm{e}}$ Meeting de Médecine et Traumatologie du Sport. Liévin-Riaumont.

d'éviter au maximum les récidives, un retour prématuré au sport entraînant de nouvelles rechutes. Les avancées ingénieuriques des échographes permettent aujourd'hui d'observer plus précisément l'aspect architectural et lésionnel musculaire :

- angle de pennation, interpénétration conjonctive, aire de section etc. ;

- meilleure définition de la localisation des lésions.

La lésion musculaire intrinsèque devrait être décrite comme une lésion myo-aponévrotique par mouvement excentrique et/ou overstretching, correspondant à une atteinte par désinsertion (Bruchard, 2008). Elle comprend une atteinte de la structure musculaire, une atteinte de la structure conjonctive, ou une atteinte de l'intimité myoconjonctive. Au niveau de l'intimité muscle-aponévrose des protéines agrafes, dites de liaison, ont été découvertes permettant l'adhésion des éléments micro comme macroscopiques entre eux. Trois zones de faiblesse sont distinguées lorsque la fibre se décolle de sa cloison centrale (ou périphérique); une lésion myo-aponévrotique centrale (périphérique) se situe dans le corps musculaire alors qu'une lésion myotendineuse atteint la zone de jonction tendino-musculaire (Brasseur, Bach, Renoux, \& Zitoun-Eiss, 2010).

La lésion myo-aponévrotique survient principalement en excentrique (Kaux, et al., 2013). D'après la courbe force/vitesse de raccourcissement des fibres de Komi, le travail excentrique correspond à un travail frénateur protecteur de l'articulation (Middleton, \& Montero, 2004). Les tensions développées en excentrique sont supérieures au concentrique. Cet allongement du muscle implique la sollicitation du tissu contractile, mais surtout provient de la résistance du tissu conjonctif de soutien. Le muscle est formé de deux biomatériaux qui sont régis par les lois de l'ingénierie : la compliance, la viscoélasticité, le fluage etc. Le coefficient de viscoélasticité de Kelvin Voigt ne permet pas l'allongement si les capacités viscoélastiques sont dépassées mais entraîne la rupture des biomatériaux (Bruchard, 2010). Lors de l'étirement maximal et rapide du complexe ou d'une puissante contraction excentrique, le point de rupture interviendra au point d'intimité myo-conjonctive.

L'objectif de la rééducation des lésions musculaires chez le sportif est de récupérer une mobilité et une motricité compatibles avec la reprise de son activité ainsi qu'une récupération totale de la force concentrique et surtout excentrique du groupe musculaire lésé.

À la suite d'une lésion musculaire, le processus de réparation se déroule en trois phases : phase inflammatoire (J0 à J4), la phase de reconstruction cellulaire ou phase proliférative (J3 à J14) et la phase de remodelage (J14 à J60) (Fig. 1). La phase inflammatoire est caractérisée par la formation d'un hématome et la nécrose de l'extrémité des fibres lésées. Les macrophages éliminent les débris cellulaires et participent à la production de cytokines et de facteurs de croissance favorisant la seconde phase. La phase de réparation débute dès la 
$24^{\mathrm{e}}$ heure post traumatique par l'activation, la migration et la prolifération des cellules satellites de Mauro. En fonction du milieu dans lequel elles se trouvent l'importance du tissu cicatriciel sera modifiée. En effet, la concentration d'IGF-1, favorise leur transformation en myoblaste et celle de TGF $\beta$ favorise la formation de fibrose. À partir de J3, un tissu de granulation sera produit, des protéoglycans et du collagène de type 3 seront synthétisés par les myofibroblastes (différenciation des cellules de Mauro) pour former le tissu cicatriciel. À partir de J5, les myoblastes s'organisent et s'alignent en myotubes, les cellules musculaires en régénération vont pénétrer le tissu cicatriciel, des molécules d'adhésion (intégrines) seront exprimées sur les bords de chaque myofibre ancrant ainsi les bords du tissu cicatriciel au tissu avoisinant.

Entre J10 et J15, le site lésionnel est infiltré par des fibroblastes déposant une matrice fibreuse dense. Le processus de cicatrisation est alors le résultat d'une compétition entre régénération et développement de fibrose dont l'issue conditionne la qualité de la guérison et la survenue de limitations fonctionnelles et/ou douleurs résiduelles. De J14 à J21, le tissu cicatriciel se condense, diminue de taille, les myofibrilles nouvellement formées continuent de pénétrer la zone cicatricielle (Järvinen, Järvinen, Kääriäinen, Kalimo, \& Järvinen, 2005). La cicatrisation du tissu musculaire nécessite une bonne vascularisation de la zone lésée afin de favoriser la synthèse de nouvelles fibres musculaires, la réalisation de traction axiale afin d'orienter la cicatrisation et le respect de l'innervation qui par son activité va favoriser la différenciation cellulaire. Dans les suites d'une lésion myoaponévrotique, le travail excentrique prend toute sa place et permet :

- l'activation des cellules satellites de Mauro (Middleton, Duprey, Boudine, \& Delest, 2005),

- la stimulation et le renforcement de l'armature conjonctive (Allbrook, 1981),

- le bon alignement des fibres de collagène (luttant contre la fibrose et la calcification par la structuration des espaces) (Middleton, et al., 2013),

- le renforcement à l'étirement lésionnel du complexe musculo-tendineux (Middleton, et al., 2013),

- l'activation de la synthèse protéique responsable de la myofibrillogenèse, permettant le recollement de l'interface myo-aponévrotique, assurant ainsi une solidité à la cicatrice (De Deyne, 2001).

Pour réaliser ce travail excentrique, l'isocinétisme nous semble être un moyen idéal car il permet un travail précis et adapté sécurisé en amplitude (constante par un système électronique complété par des butées mécaniques), en intensité (biofeedback visuel via écran, limitateur de couple de force) et en vitesse. Dès le troisième jour post-lésionnel, des étirements passifs doux en course moyenne peuvent être réalisés vu que l'étirement passif est la tension minimale du travail excentrique (Croisier, Maquet, Codine, \& Forthomme, 2008). Notre protocole isocinétique comporte le premier jour, une évaluation initiale de la force musculaire du côté sain, en mode excentrique, aux mêmes vitesses angulaires que celles utilisées par la suite au cours de la rééducation : 5, 10, 15 jusqu'à 30\% (Queiros Da Silva, Cotte, Vicard, \& Chantelot, 2005).

- La règle de la non-douleur est fondamentale tout au long de la rééducation.

- Chaque séance est précédée d'un échauffement de 10 min sur bicyclette à 60 watts et suivie d'une récupération sur bicyclette associée à une kinésithérapie manuelle classique (cryothérapie, physiothérapie anti-inflammatoire, étirements musculo-tendineux passifs progressifs, crochetage myo-aponévrotique, travail excentrique manuel et proprioception).

- Sollicitations excentriques débutées sur un mode cicatriciel $(5 \% \mathrm{~s})$, sur une amplitude non douloureuse de $45 \%$ de l'amplitude maximale, à une intensité de $20 \%$ du MFM du groupe musculaire homologue.

- Application du protocole de Stanish adapté aux lésions musculaires (Stanish, Rubinovitch, \& Curwin, 1986). Nous intensifions progressivement les contraintes (5 à $10 \%$ à chaque séance), l'amplitude jusqu'à la course externe et la vitesse jusque $30 \%$ s.

La progression dans les vitesses angulaires se réalise lorsque le moment de force atteint $70 \%$ du moment de force du groupe homolatéral à vitesse identique. Notre expérience de la lésion myo-aponévrotique nous a montré que ce protocole pouvait s'appliquer quotidiennement, voir bi-quotidiennement la deuxième semaine post lésionnelle sans dépasser un nombre maximal de répétitions de 50-60 par jour. Généralement, un protocole de quatre séries de 6-8 répétitions par séance est appliqué et très peu de douleurs retardées (Delayed Onset Muscle Soreness : DOMS) avec ce type de travail sont rapportées. Naturellement chez un sportif de haut niveau, une adaptation au jour le jour est nécessaire en fonction de l'état de fatigue, de la douleur, des courbatures de la préparation physique quand celle-ci a été reprise. La reprise de la course est autorisée lorsque le moment de force en excentrique atteint $70 \%$ de la valeur du groupe musculaire homologue à $5 \% \mathrm{~s}$, des entraînements et de la compétition à $80 \%$ et $90 \%$ à $30 \% \mathrm{~s}$ (Queiros Da Silva, 2004). Ferret (2010) a réalisé une étude rétrospective sur les lésions musculaires d'ischiojambiers. Les patients consultent tardivement (deux mois en moyenne) après échec de repos sportif seul ou de la prise en charge médicale classique. Le repos seul est inefficace et les thérapies conventionnelles souvent insuffisantes (physiothérapie, crochetage, stretching...). Tout retard de prise en charge augmente le risque de séquelles et de récidives. Nous rappelons donc l'importance du diagnostic et du traitement précoce des lésions musculaires. Cette étude nous montre qu'un travail excentrique précoce IJ (J6) est possible et permet de récupérer $90 \%$ de la force excentrique du groupe homologue dès J21. 
Les effets néfastes de l'immobilisation sont bien connus : augmentation du nombre de fibres lésées, orientation anarchique, atrophie musculaire, baisse de résistance à la traction (Järvinen, \& Lehto, 1993). Laisser un sportif au repos suite à une lésion musculaire est donc contraire aux bonnes pratiques. Enfin, la prise en charge d'un sportif de haut niveau blessé débouche inévitablement sur la problématique de la programmation du retour sur le terrain. Une réponse pertinente devrait s'appuyer sur l'analyse (localisation, gravité) et les conséquences de la blessure, du type de discipline pratiquée et des facteurs de risques lésionnels ou d'induction de pathologies secondaires. La réalité de terrain démontre cependant des attitudes très contrastées et la régulière absence de critères objectifs basés sur des tests, mesures et/ou examens complémentaires (Delvaux, Rochcongar, Ferret et al., 2013).

Sur le plan scientifique, la littérature consacrée au « return to play criteria » apparaît également pauvre et souvent basée sur des facteurs hypothétiques plutôt qu'issues de l'« evidence based medicine » (Delvaux, Rochcongar, Bruyere, et al., 2013). Toutefois, Croisier, Ganteaume, Genty, Binet, \& Ferret (2006) ont étudié sur une série de plus de 600 footballeurs professionnels les performances isocinétiques des fléchisseurs et extenseurs du genou mesurées en début de saison et les a confrontées aux antécédents lésionnels des structures (osseuses, ligamentaires, méniscales, musculo tendineuses) à proximité du genou. L'ensemble de ces joueurs explorés étaient jugés aptes à la pratique compétitive par le staff médical du club au moment des tests isocinétiques. Plus de $60 \%$ des joueurs explorés rapportaient des antécédents lésionnels significatifs et, parmi ceux-ci, $65 \%$ conservaient des insuffisances et des déséquilibres de performances musculaires. Cette observation permet de s'interroger sur les critères utilisés dans le football professionnel pour définir le moment le plus opportun au retour compétitif. Delvaux et al. (2013) ont récemment déterminé les critères objectifs devant être remplis avant d'autoriser un sportif blessé aux IJ à reprendre l'activité compétitive sans restrictions. Ces critères sont : absence de douleurs, récupération d'une force musculaire appropriée, un test spécifique au sport, les sensations subjectives du sportif, la souplesse musculaire, l'imagerie médicale, la correction de dysfonctions lombaires et/ou sacro-iliaques.

Au-delà de compromettre certaines composantes de la performance sportive, les anomalies et déséquilibres de force musculaire majorent le risque de survenue de lésions musculaires intrinsèques des ischio-jambiers (Croisier, 2004, 2005 ; Croisier, Forthomme, Namurois, Vanderthommen \& Crielaard, 2002). Les joueurs de football professionnel qui présentent, en début de saison, des anomalies de certains paramètres isocinétiques (particulièrement en mode excentrique) possèdent un risque de déchirure des ischio-jambiers multiplié par 4,5 en comparaison avec des joueurs équilibrés (Croisier, Ganteaume, Genty, Binet, \& Ferret, 2008). Les joueurs, initialement déséquilibrés qui bénéficient d'un programme de compensation efficace, présentent une réduction significative du risque lésionnel. À ce jour, il semble légitime de recommander un contrôle des performances musculaires lors du retour sur le terrain après certaines blessures chez le sportif.

L'intérêt préventif de tests en début de saison, visant à identifier et compenser par un renforcement excentrique des sujets à risque de lésion d'ischio-jambier a été démontré :

- Pour certains groupes musculaires comme les IJ, le renforcement excentrique en prévention demeure essentiel (Thorborg, 2012). L'entraînement excentrique des IJ, à vocation frénatrice, dans une position d'allongement va permettre de repousser les limites de viscoélasticité du muscle qui se traduira par un déplacement de la courbe tension/longueur de celui-ci vers la droite (Brughelli, \& Cronin, 2007; Proske, \& Morgan, 2001). Ainsi une augmentation du nombre de sarcomères en série va permettre au muscle de développer sa tension maximale dans la position où il est le plus sollicité (théorie de Goldspink) (Williams, \& Goldspink, 1971). Les fibres musculaires seront plus grandes et l'angle de pennation plus fermé, permettant ainsi une configuration architecturale optimale (Proske, \& Morgan, 2001).

- Durant les saisons 1999 et 2000, en Islande et en Norvège, l'incidence des blessures a été relevée puis, en 2001 un programme comportant des exercices d'échauffement, de souplesse et de force introduit (Arnason, Andersen, Holme, Engebretsen, \& Bahr, 2008). Cette prise en charge a permis une diminution de $65 \%$ des blessures des IJ sans incidence sur le taux de récidive. Le travail de renforcement excentrique comportait des « Nordic Hamstring Lowers ».

- Dans une étude plus ancienne, Brockett, Morgan et Proske (2001) avaient fait effectuer le même type d'exercices à des sujets en mesurant leur force isocinétique excentrique. Cet auteur s'était rendu compte que le travail excentrique déplaçait de quelques degrés le pic de force vers la droite, par une augmentation du nombre de sarcomères en série via le principe de Goldspink. Ainsi les ischio- jambiers pouvaient produire plus de force à un degré d'allongement supérieur, ce qui, de la sorte, pouvait leur conférer une meilleure résistance à l'étirement lésionnel et éviter la survenue de lésions.

En résumé, les lésions musculaires demeurent un problème majeur en traumatologie du sport. Elles sont à l'origine d'un absentéisme important chaque saison pour plusieurs joueurs d'une même équipe. Un programme de réadaptation cohérent et de prévention sur certains groupes musculaires, insistant sur le travail excentrique, permet d'en diminuer l'incidence et le taux de récidive. 


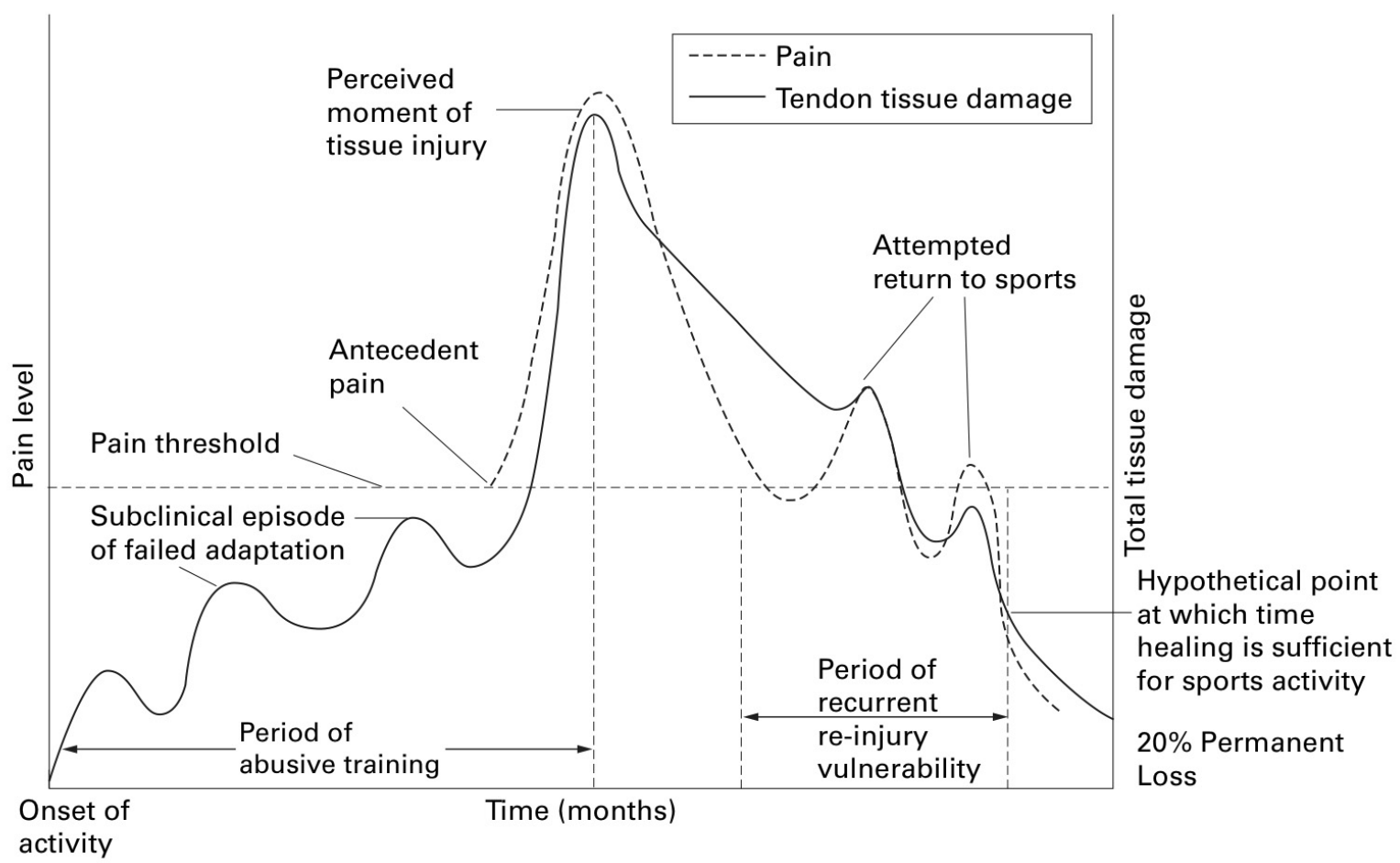

Fig. 2. Théorie de «l'iceberg » de la tendinopathie d'après Leadbetter et al. (1992), d'après Khan, Maffuli, Coleman, \& Cook (1998).

\section{Pathologies tendineuses}

Contrairement aux apparences, « la tendinite » présente un pronostic bien souvent incertain. Actuellement, le syndrome clinique associant une douleur progressive et un épaississement du tendon et/ou du péri tendon, qui entraîne une réduction de la performance doit être dénommé 《tendinopathie » et/ou « paratendinopathie » (Kaux, Forthomme, et al., 2011). La physiopathologie des tendinopathies correspond à un processus multifactoriel, faisant intervenir des facteurs intrinsèques et extrinsèques, évoluant de manière indépendante ou en combinaison (Kaux, Forthomme et al., 2011). En particulier, il semblerait que des charges mécaniques répétitives et/ou l'application d'une charge dépassant la résistance du tendon pourraient entraîner progressivement des lésions micro et macroscopiques. Les fibres de collagène commencent à se dénaturer causant progressivement la tendinopathie pouvant entraîner une rupture partielle voir totale du tendon (Kaux, Forthomme, et al., 2011). On retrouve ainsi une fragmentation du collagène avec un accroissement du collagène III, une production augmentée de proteoglycans, une apoptose des ténocytes, ainsi qu'une réaction angioblastique avec néo vascularisation et néo innervation pouvant entraîner des douleurs chroniques (Bard, 2012; Kaux, Forthomme, et al., 2011). D'autres modifications sont décrites, telles que la présence de glutamate, de lactate, de substance P et d'autres peptides. Une meilleure connaissance de l'anatomopathologie de ces lésions nous mènera à une démarche thérapeutique plus efficace.

Les tendinopathies apparaissent fréquentes au sein de la population active et sportive à dominance de course à pieds, de sauts (football, basket-ball, volley-ball) (Kaux, Forthomme, et al., 2011). Dans ces sports, par exemple, la prévalence de la tendinopathie rotulienne est estimée à $14,2 \%$, mais son incidence chez des joueurs professionnels de volley-ball peut aller jusqu'à $40 \%$ (Lian, Engebretsen, \& Bahr, 2005). Le tendon est un tissu conjonctif différencié, très peu vascularisé, reliant l'os au muscle, avec lequel il forme un couple indissociable sur le plan fonctionnel (Bruchard, \& Duée, 2008). Sa structure, en particulier sa grande richesse en fibres de collagènes, est étroitement adaptée aux forces de traction avec un effet d'amortissement entre le muscle, organe moteur, et la pièce squelettique, organe effecteur.

Leadbetter (1992) ont démontré comment le tendon subissait des lésions infra cliniques, avec tentatives de réparation et d'adaptation, pour aboutir à un stade symptomatique qui conduit le patient à consulter (Fig. 2). La difficulté pour le thérapeute est de juger le moment où la réparation est suffisante pour une reprise de l'activité sans risque de rechute, malgré la persistance d'un déficit de résistance de $20 \%$. L'adaptation physiologique du tendon correspond à la capacité du corps à réparer les microlésions tissulaires subies suite à un effort. Cette adaptation physiologique sera notamment conditionnée par une charge adéquate, un état nutritionnel optimal, 


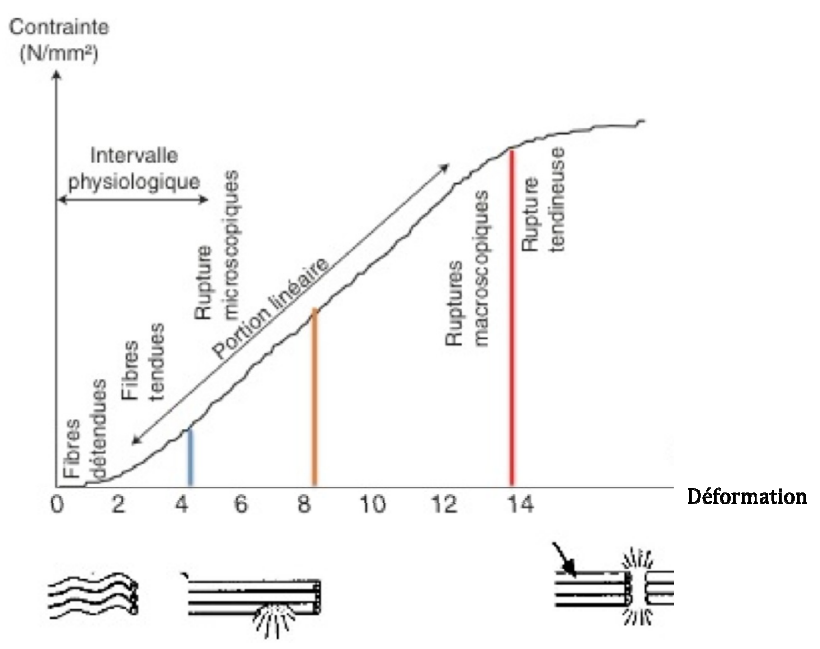

Fig. 3. Courbe tension/déformation du tendon d'après Khan, Maffuli, Coleman, \& Cook (1998).

un matériel adapté (Kaux, Forthomme, et al., 2011). Si ces conditions ne sont pas respectées, la réparation physiologique sera insuffisante et le tendon entrera en phase de désadaptation.

Prenons l'exemple d'un homme de $70 \mathrm{~kg}$, qui réalise un jogging à allure modérée. La contrainte exercée sur le tendon d'Achille à chaque foulée est estimée à 6000 Newtons (N). La courbe tension/déformation d'un tendon démontre qu'une élongation répétée de 5 à $6 \%$, correspondant approximativement à des contraintes de $6000 \mathrm{~N}$, est susceptible d'induire des micro-ruptures intra tendineuses (Fig. 3). L'atteinte tendineuse micro traumatique découle d'une surcharge tensionnelle du complexe musculo-tendineux entraînant une incapacité à la réparation tendineuse (El Hawary, Stanish, \& Curwin, 1997; Khan, Maffuli, Coleman, Cook, \& Taunton, 1998; Rees, Wolman, \& Wilson, 2009). Les stratégies thérapeutiques anti-inflammatoires apparaissent peu efficaces mais peuvent être utilisées en phase aigue durant un temps très court afin de diminuer la douleur et permettre une rééducation excentrique le plus rapidement possible (Kaux, Forthomme, et al., 2011). Actuellement, le traitement conservateur des tendinopathies s'avère complexe. Parmi les techniques décrites, le traitement passif, peu efficace sur le risque de récidive, propose essentiellement le repos, la glace et l'électrothérapie afin de limiter la douleur (Kaux, Forthomme, et al., 2011). Les approches thérapeutiques actives, plus actuelles et adaptées à l'étiopathogénie, cherchent à améliorer la qualité de résistance tensionnelle du tendon par l'application de programme actif. La structure tendineuse doit aussi bénéficier d'un entraînement adapté visant à améliorer ses qualités mécaniques et le protéger des microlésions résultant des contraintes extérieures. En 1986, les travaux de Stanish et al. (1986) introduisent le concept du programme excentrique. Après six semaines d'un travail excentrique sur 200 patients souffrant d'une tendinopathie rotulienne chronique, Stanish note une disparition complète des douleurs chez $44 \%$ des patients et une diminution marquée des plaintes pour $43 \%$ des sujets. À la suite de ce premier travail innovant, l'efficacité de la rééducation excentrique apparait régulièrement démontrée dans la prise en charge conservatrice des tendinopathies.

L'exercice intègre les paramètres d'allongement résisté de l'unité musculo-tendineuse (selon une amplitude de mouvement définie), la vitesse du mouvement et l'intensité de la contraction (Forthomme, Kaux, Crielaard, \& Croisier, 2011). Les modalités d'application du protocole conditionnent son efficacité : vitesse lente et intensité sous-maximale au stade initial, ces deux paramètres étant progressivement intensifiés en respectant strictement l'indolence : le débattement articulaire sélectionné intégrera dès que possible le secteur d'allongement maximal (piste externe). Un minimum de 20 à 30 séances de traitement, à raison de trois fois par semaine semble nécessaire pour une efficacité optimale. La fréquence de trois séances par semaine autorise une période de repos suffisante dans la phase de remodelage caractérisée par l'augmentation des ponts de collagène.

Pour les nombreux avantages qu'offrent l'isocinétisme, Croisier et al. (2001) ont étudié l'effet du traitement excentrique chez 34 patients atteints de tendinopathies chroniques (9 tendinopathies achilléennes, 10 rotuliennes et 15 épicondylites). Les progrès du traitement excentrique s'objectivent, dans cette étude, par les progrès sur l'échelle visuelle analogique (EVA), par la mesure de la force musculaire isocinétique développée en fin de traitement (en comparaison bilatérale en concentrique et excentrique), par la modification de l'image échographique et par l'analyse des conditions de retour à l'activité sur le terrain. Après 10 séances, une réduction significative de l'EVA de 7 à 5 en moyenne pour les trois types de tendinopathies. À la vingtième séance, l'EVA est de 2,5 en moyenne. À la trentième séance, l'évaluation isocinétique de force maximale ne montre pas de différence bilatérale par rapport au côté sain. L'échographie montre une structure tendineuse homogène et normalisation des diamètres tendineux pour $40 \%$ des 34 patients.

Dans le traitement des tendinopathies achilléennes et rotuliennes, Croisier et al. (1999) utilisent des vitesses de travail excentrique relativement rapides de $30 \%$ s à $120 \%$ s. La possibilité d'utiliser des vitesses de travail excentrique plus lentes de $5 \%$ s à $30 \%$ s a également été décrite (Queiros Da silva, Cotte, Vicard, Chantelot, \& Ferret, 2005). Nous utilisons depuis des années des vitesses excentriques lentes dites « cicatricielles », elles ont l'avantage de ne pas déclencher de DOMS, de permettre un temps de travail excentrique beaucoup plus long, et de donner de très bons résultats (Queiros Da silva, Cotte, Vicard, Chantelot, \& Ferret, 2005). Ceci explique vraisemblablement un nombre de répétitions plus important dans les protocoles d'application du travail isocinétique excentrique de Croisier et al. (2008). Enfin, le traitement excentrique montrerait de meilleurs résultats à court terme 
qu'en concentrique sur une période de 12 semaines (Mafi, Lorentzon, \& Alfredson, 2001). Le travail excentrique entraînerait plus de remaniements intra tendineux que l'exercice concentrique (Kaux, Forthomme, et al., 2011; Kaux, Drion, \& Croisier, 2011; Mouraux, \& Brassinne, 2009, 2011). Le travail excentrique agirait par le mécanisme de mécano transduction c'est-à-dire qu'il convertirait la charge mécanique en réponse cellulaire, ce qui entraînerait à long terme des modifications de la structure tendineuse et aboutirait à une modification des propriétés mécaniques du tendon (Landberg, et al., 2007). Landberg et al. (2007) montrent qu'après 12 semaines d'entraînement excentrique la douleur est fortement réduite et surtout stimule la synthèse de collagène de type I (plus résistant aux forces de traction) mais sans modifier le taux de dégradation de celui-ci (Landberg et al. 2007). Enfin, Kaux et al. (2013) ont comparé dernièrement les effets de deux méthodes d'entraînement sur des tendons de rats. Au terme de ce travail, ils ont démontré que l'effort excentrique entraînait une augmentation plus importante de masse musculaire, une amélioration de la résistance à la traction longitudinale que l'effort concentrique. Cette étude confirme donc que les propriétés mécaniques du tendon de rat sont améliorées par le travail excentrique. Le tendon, plus résistant, augmenterait sa quantité de fibres de collagène et probablement les interactions entre elles.

Actuellement les tendinopathies demeurent un défi thérapeutique car certaines évoluent selon un nombre désespérément chronique en raison de leur caractère rebelle aux différentes thérapeutiques conservatrices classiques (anti-inflammatoires, rééducation, ondes de chocs...). Les infiltrations de plasma riche en plaquettes (PRP) pourraient représenter un espoir thérapeutique suite aux résultats de diverses expérimentations in vitro et animales. L'adjonction de sollicitations excentriques dans les suites de l'injection de PRP améliore l'efficacité de ce type de traitement (Kaux, \& Crielaard, 2013).

En résumé, les traitements en mode excentrique ont une place à part entière dans la prise en charge des tendinopathies. Les mécanismes d'action sont actuellement méconnus mais différentes hypothèses d'origine mécanique, neurovasculaire ou biochimique ont vu récemment le jour (Mouraux, \& Brassinne, 2011). Une meilleure connaissance des effets de l'excentrique sur la cicatrisation tendineuse permettra probablement d'affiner les protocoles de rééducation.

\section{Rupture du ligament croisé antéro-externe (LCAE)}

Les entorses du genou liées à la pratique du football sont fréquentes et demeurent problématiques, indépendamment du sexe et du niveau de jeu. La blessure qui attire le plus l'attention est la rupture du LCAE qui entraîne une longue absence des terrains et peut, même, mettre fin à la carrière du joueur. Les blessures au LCAE sont plutôt rares dans le football d'élite où elles représentent moins de $1 \%$ des blessures occasionnant une absence des terrains (Walden, Hägglund, Magnusson, \& Ekstrand, 2011). Sur le plan statistique, une équipe d'élite de 25 joueurs peut s'attendre à une blessure au LCAE toutes les deux saisons. La quasi-totalité des joueurs présentant une rupture complète du LCAE ont été traités chirurgicalement, reflétant l'opinion générale des médecins du football sur la nécessité d'une chirurgie de reconstruction afin de pouvoir continuer la pratique du football. En effet, Baudot (2012) conclue que le traitement orthopédique pourrait être proposé uniquement à une population ayant une faible demande d'activités avec pivots et contacts. La pratique d'activité sportive en pivot-contact avec des mécanismes à haute énergie est souvent incompatible avec une lésion du LCAE et conduit, la plupart du temps, à un geste de réparation chirurgicale en raison du risque d'instabilité. Le risque d'instabilité du genou a ainsi donné la priorité au traitement chirurgical. L'objectif de la chirurgie est de remplacer le LCAE par un transplant dont le choix a été la source de nombreuses controverses. Les transplants tendineux ont aujourd'hui la meilleure place. L'étude de Walden et al. (2011) montre que $94 \%$ des joueurs ayant subi une opération après une blessure au LCAE ont repris complètement au niveau d'élite dans les 10 mois suivant l'opération et $89 \%$ ont participé à un match d'élite dans les 12 mois. Ces résultats contrastent avec des données présentées sur les joueurs amateurs, où seuls $53 \%$ à $65 \%$ étaient en mesure de jouer deux ans après ligamentoplastie (Dauty, Le Brun, et al., 2008).

Les techniques de réparation arthroscopiques du LCAE regroupent actuellement les techniques de reconstruction isolée du LCAE le plus souvent réalisées par des autogreffes (tendons rotulien, de la patte d'oie ou du fascia-lata) et des techniques de reconstruction associant plastie intra-articulaire et extra-articulaire. Cette plastie externe présente le but de renforcer la stabilité rotatoire du genou. La rééducation post opératoire est fondamentale. Pour les sportifs de haut niveau, elle est optimisée temporellement et quantitativement dans le respect des délais thérapeutiques cliniques. La prise en charge quotidienne, l'expérience du médecin rééducateur et du kinésithérapeute du club, sont vraisemblablement l'une des raisons du retour à la compétition dans le football professionnel (Ekstrand, 2011). Les objectifs de la rééducation sont les suivants : récupérer la mobilité du genou, restaurer progressivement la force musculaire du quadriceps et des ischio-jambiers. L'important est de respecter le cahier des charges qui varie en fonction du greffon utilisé et des habitudes des équipes soignantes. Sachant que la rééducation prend une part importante dans le résultat fonctionnel après ligamentoplastie, le travail de rééducation et le travail musculaire doivent tenir compte (Middleton, Moreau, Petit, \& Montero, 2010) :

- de la fragilité du montage chirurgical les premières semaines ; 
- des phénomènes de ligamentisation;

- de l'état du genou ;

- du profil psychologique du patient;

- des forces mécaniques qui s'exercent sur le genou lors de la rééducation (Lutz, Palmitier, \& An, 1993).

La rééducation doit également tenir compte du tendon prélevé. L'utilisation du tendon rotulien selon Kenneth-Jones (KJ) fragilise l'appareil extenseur du genou. La plastie réalisée à partir du gracile et du demitendineux (DIDT) respecte le quadriceps, mais fragilise les fléchisseurs du genou et surtout les rotateurs internes du genou. La plastie à l'aide du fascia-lata (Mac Intosh) n'est pas non plus sans inconvénient. Outre la taille de la cicatrice, le fascia-lata participe à la stabilité latérale $\mathrm{du}$ genou. Le renforcement musculaire isocinétique en chaîne cinétique ouverte (CCO) s'intègre dans le programme de rééducation, en complément des autres techniques et son utilisation suit l'évolution des différentes phases. L'introduction de l'isocinétisme est variable selon le type de plastie et l'évolution clinique du genou, après disparition des douleurs et de l'inflammation. Le renforcement isocinétique en chaîne ouverte permet un renforcement spécifique du quadriceps et des IJ mais les contraintes sur le LCAE et l'appareil extenseur sont importantes surtout proches de l'extension et au maximum entre $15^{\circ}$ et $30^{\circ}$ quelle que soit la vitesse (Bovard, Belmahfoud, \& Salvator-Witvoet, 2003). Ces contraintes s'accroissent lorsque la vitesse diminue (en concentrique) et avec l'intensité de la contraction quadricipitale. L'utilisation des systèmes « anti-tiroir » ou d'un contre appui proximal diminue les contraintes (Paulos, Wnorowski, \& Beck, 1991). Dans le renforcement musculaire, il faut donc tenir compte des contraintes sur le pivot central du genou.

Dans une étude rétrospective multicentrique, Condouret et al. (2008) retrouvent à deux ans d'une ligamentoplastie (KJ ou DIDT), un déficit résiduel moyen de $10 \%$ sur les extenseurs, non prélèvement dépendant, et un déficit moyen des fléchisseurs de $10 \%$ significativement supérieur dans le groupe des plasties aux IJ, en relation avec le nombre de tendons prélevés : $-7 \%$ lorsqu'il n'y a aucun prélèvement, $7 \%$ avec prélèvement d'un tendon et $17 \%$ avec deux tendons prélevés. La relation entre le niveau de récupération musculaire du quadriceps et des ischio-jambiers à deux ans et la qualité du résultat fonctionnel incitent, devant ce déficit significativement supérieur des fléchisseurs au décours des plasties aux IJ, à modifier nos programmes de rééducation actuels et à mettre un accent particulier sur la rééducation précoce des ischio-jambiers en mode excentrique. Celle-ci doit débuter dès les premières semaines post opératoires, la prise de greffon se révélant un équivalent de désinsertion musculaire.

\subsection{Plastie DIDT}

Dans les suites d'une ligamentoplastie du genou, le renforcement musculaire est principalement orienté sur les ischio-jambiers. C'est la seule structure active qui peut protéger la plastie et éviter qu'elle se détende. Cependant lors de la rééducation du DIDT, les différents auteurs contre-indiquent le travail au niveau de ces muscles pendant trois à six semaines post opératoires. Or, la mise au repos des IJ, n'a pas fait la preuve de la réduction des complications au niveau du site donneur. Cette attitude protectionniste non justifiée est contraire à tous les travaux sur la cicatrisation des tissus mous. Fabri et al. (2007) ont montré que le renforcement d'emblée des fléchisseurs du genou n'est pas préjudiciable puisque le taux d'épisodes à la face postérieure de cuisse $(12,5 \%$ dans leur étude) est inférieur à celui évalué dans la littérature où ils sont souvent supérieurs à $20 \%$. Puig, Trouve, Savalli, Laboute et Aucagne (2005) ont proposé également un réveil musculaire du site donneur. Ils sollicitent également les muscles du site donneur au septième jour post opératoire par des contractions statiques entre $45^{\circ}$ et $90^{\circ}$ de flexion. Son action reste prudente puisque la charge à 28 jours est de 1 à $3 \mathrm{~kg}$ à $\mathrm{J} 45$.

Denais, Cornet, Guyard et Dauty (2010) s'est également inspiré du travail excentrique précoce des IJ de Fabri et al. (2007). En effet, dans leur protocole post ligamentoplastie, ils proposent un travail manuel des IJ jusqu'à J21 afin d'avoir une meilleure protection du site donneur et apprécier les capacités post opératoires du patient. Le passage au renforcement instrumentalisé ne se fait qu'à J21, date à laquelle le tissu conjonctif cicatriciel possède déjà un minimum de résistance. Par ailleurs, leur protocole utilise des résistances adaptées au pourcentage de la charge maximale freinée côté sain, afin d'adapter la rééducation aux capacités physiques initiales du patient. Il est admis aujourd'hui l'effet délétère de la prise de greffe sur les IJ lors de la technique du DIDT. Dans le sport de haut niveau, vouloir optimiser la rééducation paraît séduisant. En effet, nous pensons que plus la récupération des IJ sera rapide, plus vite leur rôle frénateur sera obtenu, ce qui permettra également de gagner du temps sur les autres programmes de la rééducation. Ainsi, dès que les IJ seront compétents, le travail proprioceptif dynamique en charge, préconisé dans les préventions primaire et secondaire, pourra être débuté. Nous proposons un travail excentrique isocinétique des IJ dès J10 post-opératoire (lorsque la flexion atteint $90^{\circ}$ ) dans l'axe flexion/extension du genou (Barthélémy, \& Ferret, 2010). À partir de J30, nous sollicitions le site de prélèvement dans sa composante rotatoire. Nous pensons que le travail excentrique des IJ dans leur fonction de rotateurs du genou peut, du fait de son effet « protecteur articulaire », améliorer la stabilité du genou et éviter à long terme la déstabilisation rotatoire du genou opéré. Dans la rééducation, il est important d'alterner le travail des IJ dans leur fonction de fléchisseurs et de rotateurs du genou afin de les solliciter dans leurs deux composantes d'action. À J60, nous considérons que le site de prélèvement est bien cicatrisé. Nous débutons alors le travail isocinétique des IJ en concentrique à des vitesses 
angulaires de $180 \%$ s à $60 \%$ s (progressivement en pyramide inversée) sur une amplitude articulaire comprise entre $10^{\circ}$ et $100^{\circ}$ de flexion. Le retour à l'extension s'effectue pour le quadriceps à une vitesse angulaire rapide de $240 \%$ s̀ $180 \%$ s, en développant une force très largement sous maximale sur la même amplitude articulaire. Dans ces conditions, nous ne sommes pas délétères pour la plastie.

En résumé, dans la prise en charge des ligamentoplasties de type DIDT, nous nous inspirons fortement des principes de prise en charge des désinsertions myoaponévrotiques, à savoir un travail excentrique sous maximal à vitesse lente des IJ avec tous les avantages que peut comporter l'isocinétisme.

\subsection{Plastie KJ}

Si le transplant libre au tendon rotulien fragilise la rotule et le tendon rotulien, la qualité de la rééducation peut réduire les incidents liés au prélèvement (Fichez, Middleton, Duprey, Boudine, \& Delest, 2003). En fait, le réel problème posé par le prélèvement aux dépens de l'appareil extenseur est la survenue d'une tendinopathie rotulienne qui peut devenir invalidante lors de la pratique de sports de sauts. Nous préconisons alors un travail excentrique sous maximal des extenseurs du genou avec dispositif anti tiroir très doux à $\mathrm{J} 60(5 \% \mathrm{~s}$ sur une amplitude limitée de $30^{\circ}$ en extension en respectant 50 à $100 \mathrm{Nm}$ ) afin d'améliorer la cicatrisation du tendon rotulien dont la chirurgie a prélevé un tiers. Les fléchisseurs du genou sont débutés très tôt en concentrique et excentrique.

\subsection{Renforcement du quadriceps}

Le rôle prépondérant de la musculature pour la stabilité fémoro-tibiale dynamique est unanimement accepté. Le traitement chirurgical des lésions du pivot central génère des inhibitions neuromusculaires auxquelles s'ajoute une réelle amyotrophie quadricipitale, conséquence de l'immobilisation relative. Les techniques de prévention de l'amyotrophie et de renforcement, adaptées et respectant l'intégrité de la plastie, constitueront des éléments essentiels de la rééducation. Dans une revue de la littérature, Huguet, Gateau et Mathias (2012) ont conclu qu'une association judicieuse d'un renforcement du quadriceps en chaîne cinétique ouverte $(\mathrm{CCO})$ et chaîne cinétique fermée $(\mathrm{CCF})$ semblerait apporter les meilleurs résultats en termes de stabilité du genou et de force musculaire.

Mikkelsen, Werner et Eriksson (2000) montrent que six mois après opération du LCAE, les sujets ayant effectué la rééducation incluant des exercices en CCO et $\mathrm{CCF}$ ont significativement plus développés la force du quadriceps que les sujets n'ayant effectué que de la CCF. Cependant, ces résultats n'ont pas pris en compte les différents types de chirurgie. Heijne \& Werner (2007) démontre une laxité plus importante suite à une greffe provenant des ischio-jambiers. L'étude de Heijne \& Werner (2007) a comparé les effets d'une rééducation intégrant précocement (quatre semaines après opération) des exercices de genou en $\mathrm{CCO}$ à ceux d'une rééducation intégrant tardivement (12 semaines après opération) de la CCO sur des sujets regroupés par type de reconstruction subie (soit prélèvement sur le ligament patellaire, soit prélèvement sur les IJ). Des évaluations de laxité antérieure ont été effectuées à trois, cinq et sept mois de l'opération. Lors d'une rééducation intégrant précocement de la chaîne ouverte, le groupe ayant subi une reconstruction du LCAE à partir des IJ avait une laxité significativement supérieure, à tout moment de $1 \mathrm{~mm}$, par rapport à une reconstruction du LCAE à partir du ligament patellaire. Concernant la chirurgie à partir du ligament patellaire, l'inclusion précoce ou tardive d'exercices en CCO ne semblait pas influencer la laxité antérieure. Toutoungi, Lu, Leardini, Catani et O'Connor (2000) ont montré que les forces s'exerçant sur la plastie varient en fonction de la vitesse de contraction isocinétique : de $350 \mathrm{~N}$ à $60 \%$ à $255 \mathrm{~N}$ à $180 \% \mathrm{~s}$. Par comparaison, un travail isométrique du quadriceps réalisé entre $35^{\circ}$ et $40^{\circ}$ génère des contraintes de $400 \mathrm{~N}$ sur le LCAE.

Sur les DIDT, aucune fixation osseuse ne fait l'unanimité. Dès les premiers jours, et ce quel que soit le mode de fixation utilisé, une ostéolyse des tunnels fémoraux et tibiaux s'opère (Buelow, Siebold, \& Ellermann, 2002). Il y aurait un élargissement des tunnels qui se stabiliserait à six mois, ce qui nous incite à rester prudents dans la sollicitation de la plastie. L'évolution histologique du transplant est lente, et sur le plan biomécanique, cela se traduit par une fragilisation de la plastie qui est maximale entre le deuxième et le quatrième mois. La résistance mécanique augmente progressivement et peut être considérée comme satisfaisante à la fin du sixième mois. Noé, Billuart, Messina et Nephtali (2010) ont rappelé les différentes phases de ligamentisation et pensent que le renforcement musculaire doit se superposer à ce phénomène. Selon ces auteurs, un travail précoce du quadriceps en CCO (à partir de la sixième semaine) ne serait pas néfaste.

Sur base de ces différentes études, nous débutons chez nos sportifs de haut niveau opérés d'une ligamentoplastie de type DIDT le renforcement du quadriceps concentrique isocinétique en $\mathrm{CCO}$ à huit semaines post opératoires avec le dispositif anti-tiroir. Nous proposons alors une progression de pyramide de Davies de la semaine 8 à la semaine 11 pour approcher une vitesse d'évaluation à la semaine 12 (Tab. 1). Nous restons au moins cinq séances consécutives sur chaque pyramide avant de changer.

Sur nos plasties au tendon rotulien, nous débutons également ce protocole de renforcement isocinétique du quadriceps mais en veillant à ne pas déclencher de tendinopathie patellaire en utilisant des vitesses lentes trop précocément. La réaction du genou prime sur la progression du travail musculaire isocinétique. Contrairement à Mikkelsen et al. (2000), nous préférons rester plus prudents dans le travail isocinétique. Ils débutent le travail du 
Tableau 1. Progression du travail isocinétique post ligamentoplastie genou de la semaine 8 à 12 .

\begin{tabular}{lllllcccccc}
\hline S8 & 300 & 270 & 240 & 210 & 180 & 180 & 210 & 240 & 270 & 300 \\
\hline S9 & 270 & & & & 150 & 150 & & & & 270 \\
S10 & 240 & & & & 120 & 120 & & & & 240 \\
S11 & 210 & & & & 90 & 90 & & & & 210 \\
\hline
\end{tabular}

quadriceps en isocinétisme à six semaines postopératoires dans les modes concentrique et excentrique avec une amplitude de genou de $90 / 40^{\circ}$ de flexion progressivement ramenée à $10^{\circ}$ jusqu'à six mois postopératoires. Ils évaluent à six mois la force isocinétique à $30 \%$ s, $120 \% \mathrm{~s}$ et $240 \% \mathrm{~s}$ dans les modes concentrique et excentrique du quadriceps ainsi que la laxitométrie antérieure du genou mesurée à l'arthromètre KT-1000. Cela peut paraître surprenant, mais cette dernière ne serait pas supérieure avec un travail isocinétique du quadriceps débuté à six semaines postopératoires. De plus ce travail permettrait un retour plus rapide au sport dans le groupe $\mathrm{CCO}+\mathrm{CCF}$ ( 7 mois versus 9,5 mois dans groupe CCF). Mikkelsen et al. (2000) mettent donc en relation l'augmentation de force isocinétique du quadriceps et le retour au sport plus rapide $\mathrm{du}$ groupe $\mathrm{CCO}+\mathrm{CCF}$.

\subsection{1 Évaluation isocinétique à trois mois post-opératoire}

L'évaluation isocinétique concentrique du quadriceps et des ischio-jambiers après plastie du LCAE paraît souhaitable à trois mois post opératoires (Croisier, Gremeaux, Maquet, Crielaard, \& Forthomme, 2009). Cette approche analytique permet de détecter le ou les groupes musculaires déficitaires et compenser efficacement un déficit touchant spécifiquement un groupe donné. Si nous avons l'accord du chirurgien, ce bilan initial sera réalisé avec un appui distal. En effet, Croisier et al. ont montré que l'évaluation isocinétique pour un positionnement proximal du contre-appui minimise le déficit résiduel du quadriceps : un déficit de $10 \%$ en proximal correspond chez les mêmes sujets à une insuffisance de $30 \%$ pour un positionnement distal (Croisier, Delcour, Lhermerout, Vanderthommen, \& Crielaard, 1995). Avec l'accord du chirurgien, et à l'image des délais appliqués pour l'évaluation concentrique, les épreuves excentriques aux ischio-jambiers seront programmées dès le troisième mois post opératoire. Ce premier bilan sans anti tiroir nous paraît indispensable afin d'adapter au mieux le processus rééducatif. Si des insuffisances majeures persistent, elles nécessiteront un renforcement de compensation. Il détermine également nos critères de reprise de course dans l'axe : déficit concentrique Q à $60 \% \mathrm{~s} \leqslant 35 \%$, déficit concentrique IJ à $60 \% \mathrm{~s} \leqslant 10 \%$ et déficit excentrique IJ à $30 \% \leqslant 10 \%$.

À 3-4 mois postopératoires d'une chirurgie de reconstruction du LCAE, un déficit de force isocinétique est attendu par rapport au genou controlatéral. Ainsi des programmes de réentraînement à base d'activités aérobies sont proposés pour diminuer le déficit de force. Dauty et al. (2006) ont étudié l'effet de la course à pied et de la bicyclette en continue du quatrième au sixième mois postopératoire, et ont constaté que ce type de réentraînement n'améliore pas significativement la force des extenseurs et fléchisseurs du genou opéré. À partir de ce constat, Dauty et al. (2010) ont donc par la suite analysé l'impact d'un réentraînement par la course à pied en interval training sur la force des fléchisseurs et extenseurs du genou à quatre et six mois postopératoires. Selon eux, la course à pied n'exercerait aucune influence sur la récupération musculaire et fonctionnelle du genou. Un programme de renforcement musculaire spécifique semble nécessaire pour améliorer la force du genou. Nos sportifs bénéficiant ultérieurement d'un renforcement isocinétique adapté individuel (sur base du bilan initial) retrouvent généralement à six mois postopératoires, des performances maximales normalisées (déficit Q à $60 \%$ s $10 \%$ et déficit concentrique IJ à $60 \%$ s $<10 \%$ et déficit excentrique IJ $<10 \%$ ). Par ailleurs, entre le troisième et sixième mois, le renforcement du genou non opéré est envisagé, afin de diminuer les facteurs de risque de lésion controlatérale.

\subsubsection{Critères de reprise sportive après ligamentoplastie}

Après ligamentoplastie du LCAE et la période de rééducation, la reprise compétitive représente un moment attendu mais délicat pour le sportif. La décision du corps médical d'autoriser un athlète à reprendre la compétition étant lourde de responsabilités, il apparaît fondamental d'évaluer le plus précisément possible l'exposition au risque de récidive ou de nouvelle lésion. Afin de mieux percevoir les critères utilisés sur le plan décisionnel, Delvaux et al. (2010) ont interrogé 36 médecins responsables de clubs professionnels de football à partir d'un questionnaire portant sur les critères de reprise sportive après ligamentoplastie. Ces médecins de club ne semblent pas baser leur décision d'autoriser un retour à la compétition uniquement sur une durée théorique postopératoire, mais plutôt selon différents critères tels la stabilité dynamique du genou dans les gestes spécifiques ou la force musculaire isocinétique. Néanmoins, il n'existe pas réellement de consensus concernant les critères les plus pertinents de reprise serait nécessaire. Définir également les limites de normalité contribuerait à faciliter la détermination du meilleur moment de retour compétitif. À partir de cette réflexion, la méta-analyse de Barber-Westin et Noyes (2011) portant sur 716 articles, est particulièrement démonstrative. Celle-ci inclut les critères suivants : recul postopératoire, mobilité articulaire, stabilité, force musculaire (IJ, Q, muscles de la hanche), différents tests 
dynamiques, capacité aérobique, tests spécifiques en fonction des sports. Seuls $20 \%$ des articles abordent au moins cinq de ces critères. La force musculaire n'est envisagée comme critère de reprise du sport que dans $9 \%$ des articles. L'épanchement et la mobilité articulaire sont étudiés dans $6 \%$ des articles. Le flexum est pour nous une contre-indication à la reprise du sport. Les tests dynamiques ( leg hop test ») sont décrits dans $6 \%$ des articles. Ces auteurs recommandent pour la reprise des sports à risque un déficit inférieur à $15 \%$ au test dynamique du membre inférieur («single hop »), une translation tibiale antérieure inférieure à $3 \mathrm{~mm}$, l'absence d'épanchement, une mobilité complète et un genou indolore.

En résumé, la rééducation post opératoire est fondamentale pour une bonne préparation à la reprise du sport. Pour les sportifs de haut niveau, la transition entre la rééducation et la reprise d'un entraînement adapté constitue une phase critique qui doit être traitée par une équipe de spécialistes. L'étude de la littérature ne permet pas d'apprécier clairement les critères de reprise du sport (Daubinet, 2012). Néanmoins, ceci assurerait à l'athlète un niveau de performance sportive et de sécurité optimal. Un genou stable, indolore, sans épanchement avec une bonne force musculaire, une bonne proprioception et une bonne préparation physique sont des critères préalables à une reprise de la compétition.

\section{Bibliographie}

Allbrook, D.B. (1981). Skeletal muscle regeneration. Muscle and Nerve, 4 (3), 234-235.

Arnason, A., Andersen, T.E., Holme, I., Engebretsen, L., \& Bahr R. (2008). Prevention of hamstring strain in elite soccer: an intervention study. Scandinavian Journal Medecine Science Sports, 18, 40-48.

Barber-Westin, S., \& Noyes, F.R. (2011). Factor used to determine return to unrestricted sports activities after anterior cruciate ligament reconstruction. Arthroscopy, 27, 1697-1705.

Bard, H. (2012). Tendinopathies : étiopathogénie, diagnostic et traitement. EMC Appareil Locomoteur, 7 (2), 1-18.

Barthélémy, Y., \& Ferret, J.M. (2010). Plaidoyer pour une prise en charge précoce des ischiojambiers après DIDT. Lettre Médecine Physique Réadaptation, 26, 20-27.

Baudot, C. Traitement orthopédique de la rupture du LCA. (2012). In Rodineau J., Besch S. (Eds.), Le ligament croisé antérieur : de la rupture à l'arthrose. $30^{\mathrm{e}}$ Journée de Traumatologie du sport et de la Pitié-Salpétrière. Elsevier Masson, Issy-Les-Moulineaux, 69-73.

Bovard, M., Belmahfoud, R., \& Salvator-Witvoet, V. (2003) Isocinétisme après ligamentoplastie du LCA. In $L C A / L C P$ nouvelles approches thérapeutiques des ligamentoplasties du genou. XI ${ }^{\mathrm{e}}$ Journée de Menucourt. Sauramps médical, Montpellier, 85-92.

Brasseur, J.L., Bach, G., Renoux, J., \& Zitoun-Eiss, D. (2010). Classification des lésions musculaires; de quoi parle-t- on?
In Sans N, Lhoste-Trouilloud A, Cohen M, Guerini H, Coudreuse JM, Catonne Y. L'imagerie en traumatologie sportive (p. 145-168). Sauramps Medical, Montpellier.

Brassinne, E., \& Mouraux, D. (2011). Programmes excentriques et tendinopathies : quels mécanismes adaptatifs. De la biomécanique aux applications thérapeutiques. In Julia M, Hirt D, Croisier JL, Codine P, Hérisson C. Tendon et jonction tendinomusculaire (p. 41-47). Elsevier Masson, Issy-Les-Moulineaux.

Brockett, C.L., Morgan, D.L., \& Proske, U. (2001). Human hamstring muscles adapt to eccentric exercise by optimum length. Medecine Science Sports Exercise, 33 (5), 783-790.

Bruchard, A. (2008). La lésion myo-aponévrotique. Profession Kinésithérapeute, 17, 39-42.

Bruchard, A. (2010). La lésion myo-aponévrotique. Kiné Actualité, 1210, 19-22.

Bruchard, A., \& Duée, T. (2008). Les lésions tendineuses : conceptualisation clinique ( $1^{\text {re }}$ Partie). Profession Kinésithérapeute, 19, 41-48.

Brughelli, M., \& Cronin, J. (2007). Altering the length-tension relationship with eccentric exercise: implications for performance and injury. Sports Medecine, 37 (9), 807-826.

Buelow, J.U., Siebold, R., \& Ellermann, A. (2002). A prospective evaluation of tunnel enlargement in anterior cruciate ligament reconstruction with hamstring: extracortical versus anatomical fixation. Knee Surgery Sports Traumatology Arthroscopy 10, 80-85.

Condouret, J., Cohn, J., Ferret, J.M., Lemonsu, A., Vasconcelos, W., Dejour, D., \& Potel, J.F. (2008). Évaluation isocinétique à deux ans de ligamentoplasties du ligament croisé antérieur au tendon rotulien ou aux ischiojambiers. Revue Chirurgicale Orthopédie et Réparation Appareil Moteur, 945, 375-S382.

Croisier, J.L. (2004). Factors associated with recurrent hamstring injuries. Sports Medecine, 34, 681-695.

Croisier, J.L (2005). Isocinétisme et prévention lésionnelle musculaire. In Muscle traumatique et mécanique (p. 173-179). Masson, Paris.

Croisier, J.L., \& Crielaard, J.M. (1996). Mise au point d'un rapport isocinétique fléchisseurs du genou/ quadriceps original. Application à une pathologie musculaire. Journal de Traumatologie du Sport, 13, 115-119.

Croisier, J.L., \& Crielaard, J.M. (1999a). Exploration isocinétique : analyse des paramètres. Annales de Réadaptation et Médecine Physique, 42, 538-545.

Croisier, J.L., \& Crielaard, J.M. (1999b). Méthodes d'exploration de la force musculaire : une analyse critique. Annales de Réadaptation et Médecine Physique, 42, 311-322.

Croisier, J.L., \& Crielaard, J.M. (1999c). Exploration isocinétique : analyse des courbes. Annales de Réadaptation et Médecine Physique, 42, 497-502.

Croisier, J.L., \& Crielaard, J.M. (2004). Expérience de l'isocinétisme dans l'encadrement sportif. Journal Traumatologie Sport, 21, 238-243. 
Croisier, J.L., Delcour, J.P., Lhermerout, C., Vanderthommen, M., \& Crielaard, J.M. (1995). Influence $\mathrm{du}$ positionnement du contre appui résistif lors de l'évaluation des ligamentoplasties du genou. In : actualités en rééducation fonctionnelle et réadaptation (p. 239-245). Masson, Paris.

Croisier, J.L., Godon, B., Giordano, F., Forthomme, B., Namurois, M., \& Crielaard J.M. (1999). Tendinites rotuliennes et achilléennes : intérêt des exercices isocinétiques excentriques. In Progrès en médecine physique et de réadaptation, $3^{\mathrm{e}}$ série (p. 212-217). Masson, Paris.

Croisier, J.L., Forthomme, B., \& Foidart-Dessalle, M (2001). Treatment of recurrent tendinitis by isokinetic eccentric exercises. Isokinetics Exercise Science, 9, 133-144.

Croisier, J.L., Forthomme, B., Namurois, M., Vanderthommen, M., \& Crielaard, J.M. (2002). Hamstring muscle strain recurrence and strength performance disorders. American Journal Sports Medecine, 30, 184-188.

Croisier, J.L., Ganteaume, S., Genty, M., Binet, J., \& Ferret, J.M. (2006). Incomplete muscle strength recovery in injured professional football Players. In Abstract book of 11Th Annual Congress of the European College of sports Science, Lausanne, 361.

Croisier, J.L., Ganteaume, S., Genty, M., Binet, J., \& Ferret, J.M. (2008). Strength imbalances and prevention of hamstring injury in professional soccer players: a prospective study. American Journal Sports Medecine, 36 (8), 1469-1475.

Croisier, J.L., Maquet, D., Codine, P., \& Forthomme, B. (2008). Renforcement musculaire et rééducation : apport de l'isocinétisme. In Kotzki N. (Eds.), Dupeyron A. Renforcement musculaire et reprogrammation motrice (p. 42-50). Elsevier Masson, Issy-Les-Moulineaux.

Croisier, J.L., Gremeaux, V., Maquet, D., Crielaard, J.M., \& Forthomme, B. (2009). Evaluation excentrique après plastie du LCA : modalités et profils. In Croisier J.L., Codine P. (Eds.), Exercice musculaire excentrique (p. 97-102), Masson, Paris.

Daubinet, G. (2012). Critères de reprise du sport après reconstruction chirurgicale du LCA. In Rodineau J., Besch $\mathrm{S}$. (Eds.), Le ligament croisé antérieur : de la rupture à l'arthrose, $30^{\mathrm{e}}$ Journée de Traumatologie du sport de la Pitié-Salpétrière (p. 113-118). Elsevier Masson, Issy-LesMoulineaux.

Dauty, M., Huguet, D., Tortellier, L., Potiron-Josse, M., \& Dubois, C. (2006). Réentraînement à l'effort entre le quatrième et le sixième mois après ligamentoplastie de genou au DIDT : comparaison de la pratique de la bicyclette et de la pratique du footing avec un groupe témoin non réentraîné. Annales de Réadaptation et Médecine Physique, 49 (5), 218-225.

Dauty, M., Le Brun, J., Huguet, D., Paumier, S., Dubois, C., \& Letenneur, J. (2008). Reprise du sport pivot-contact après reconstruction du ligament croisé antérieur : technique au tendon patellaire ou aux ischiojambiers. Revue de Chirurgie Orthopédique et Réparatrice de l'appareil moteur, 94, 552-560.
Dauty, M., Menu, P., \& Dubois, C. (2010). Effet d'un entraînement à la course à pied après reconstruction du ligament croisé antérieur. Annales de Réadaptation et Médecine Physique, 53 (3), 150-161.

De Deyne, P. (2001). Application of passive stretch and its implications for muscle fibers. Physical Therapy, 81, 819-827.

Delvaux, F., Rochcongar, P., Bruyère, O., Bouriet, G., Diverse, P., Daniel, C., \& Croisier, J.L. (2010). Critères de retour sur le terrain. In $7^{\mathrm{e}}$ Congrès du GIBL. Le LCA : de la lésion au retour sur le terrain.

Delvaux, F., Rochcongar, P., Bruyere, O., Bourlet, G., Daniel, C., Diverse, P., Reginster, J.Y., \& Croisier, J.L. (2013). Return-to-play criteria after hamstring injury: actual medicine practice in professional soccer teams. In $3^{\text {rd }}$ Congress of the European College of Sport \& Exercise Physicians, Poster, Frankfurt.

Delvaux, F., Rochcongar, P., Ferret, J.M., Kaux, J.F., Crielaard, J.M., \& Croisier, J.L. (2013). La lésion musculaire des ischio-jambiers. In Julia M., Croisier J.L., Perrey S., Dupeyron A., Hérisson C. (Eds.), Prévention des troubles musculo-squelettiques chez le sportif (p. 153165). Sauramps Medical, Montpellier.

Denais, L., Cornet, D., Guyard, E., \& Dauty, M. (2010). Rééducation accélérée après ligamentoplastie par la technique DIDT par un protocole simple et adapté à chacun. Journal Traumatologie du Sport, 27, 117-120.

Ekstrand, J. (2011). A 94\% return to elite level football after ACL surgery: a proof of possibilities with optimal caretaking or a sign of knee abuse? Knee Surgery Sports Traumatology Arthroscopy, 19, 1-2.

Ekstrand, J., Hägglund, M., \& Walden, M. (2011a). Injury incidence and injury patterns in professional football: the UEFA injury study. British Journal Sports Medecine, $45(7), 553-558$.

Ekstrand, J., Hägglund, M., \& Walden, M. (2011b). Epidemiology of muscle injuries in professional football soccer. American Journal Sports Medecine, 39 (6), $1226-1232$.

El Hawary, R., Stanish, W.D., \& Curwin, S.L. (1997). Rehabilitation of tendon injuries in sport. Sports Medecine, 24, 347-358.

Fabri, S., Lacaze, F., Roussenque, A., \& Marc T. (2007). La rééducation d'emblée des ischio-jambiers après chirurgie du DIDT. Journal Traumatologie Sport, 24, 193-199.

Ferret, J.M. (2010). Apport de l'isocinétisme en médecine du sport. In : $10^{\mathrm{e}}$ Meeting de Médecine et Traumatologie du Sport, Liévin-Riaumont.

Fichez, O., Middleton, P., Duprey, E., Boudine, M., \& Delest, M. (2003). La rupture du LCA : prise du transplant problèmes posés au médecin du sport. Médecins du sport, 5\%, 13-24.

Forthomme, B., Kaux, J.F., Crielaard, J.M., \& Croisier, J.L. (2011). Programme excentrique et tendinopathie : quels outils, quel programme? In : Julia M, Hirt D, Croisier JL, Codine P, Hérisson C. Tendon et jonction tendino-musculaire (p. 91-98.), Elsevier Masson, Issy-LesMoulineaux. 
Heinje, A., \& Werner, S. (2007). Early versus late start of open kinetic chain quadriceps exercises after ACL reconstruction with patellar tendon or hamstring grafts: a prospective randomized outcome study. Knee Surgery Traumatology Arthroscopy, 15 (4), 402-414.

Huguet, J., Gateau, L., \& Mathias, F. (2012) Rééducation d'un LCA opéré. Intérêts et risques de la mise en place d'exercices en chaîne cinétique fermée et en chaîne cinétique ouverte. Kinesithérapie Revue, 12 (131), 42-47.

Järvinen, M., \& Lehto M. (1993). The effects of early mobilisation and immobilisation on the healing process following muscle injuries. Sports Medicine, 15, 78-89.

Järvinen Tero, A.H., Järvinen Teppo, L.N., Kääriäinen, M., Kalimo, H., \& Järvinen, M. (2005). Muscle injuries: biology and treatment. American Journal Sports Medecine, 33 (5), 745-764.

Jidovtseff, B., Croisier, J.L., Mordant, B., \& Crielaard, J.M. (2005). Profil isocinétique des muscles fléchisseurs et extenseurs du genou dans une population d'athlètes sauteurs. Science et Sports, 20 (5), 304-307.

Kaux, J.F., Forthomme, B., Le Goff, C., Crielaard, J.M., Croisier, J.L. (2011). Current opinion on tendinopathy. Journal of Sports Science and Medicine,10, 238-253.

Kaux, J.F., \& Crielaard, J.M. (2013). Platelet-Rich-Plasma applicationin the management of chronic tendinopathies. Acta Orthopaedica Belgica, 79, 10-15.

Kaux, J.F., Drion, P., Croisier, J.L. (2011). Influence sur le tissu tendino-musculaire du mode de contraction en entraînement : modèle animal. In : Julia M, Hirt D, Croisier JL, Codine P, Hérisson C. Tendon et jonction tendinomusculaire, Masson, Paris, 31-40.

Kaux, J.F., Hody, S., Forthomme, B., Le Goff, C., Crielaard, J.M., \& Croisier, J.L. (2013). Le travail excentrique : aspects musculaires et tendineux. In Julia M., Croisier J.L., Perrey S., Dupeyron A., Hérisson C. (Eds.), Prévention des troubles musculo-squelettiques chez le sportif. Sauramps Medical, Montpellier, 60-69.

Kaux, J.F., Drion, P., Libertiaux, V., Colige, A., Hoffman, A., \& Nusgens, B. (2013). Eccentric training improves tendon biomechanical properties: a rat model. Journal Orthopeadic Research, 31 (1), 119-124.

Khan, K.M., \& Scott, A. (2009). Mechanotherapy: how physical therapist's prescription of exercise promotes tissue repair. British Journal Sports Medecine, 43, 247-252.

Khan, K.M., Maffuli, N., Coleman, B.D., Cook, J., \& Taunton, J.E. (1998). Patellar tendinopathy: some aspects of basic science and clinical management. British Journal Sports Medecine, 32, 346-355.

Landberg, H., Ellingsgaard, H., Madsen, T., Jansson, J., Magnusson, S.P., Aagaard, P., \& Kjaer, M. (2007). Eccentric rehabilitation exercise increases peritendinous type I collagen synthesis in humans with achilles tendinosis. Scandinavian Journal Medecine Science Sports, 17, 61-66.

Leadbetter, W.B. (1992). Cell-matrix response in tendon injury. Clinical Sports Medecine, 11, 533-578.
Lian, O.B., Engebretsen, L., \& Bahr, R. (2005). Prevalence of jumper's knee among elite athletes from different sports: A cross- sectional study. American Journal Sports Medecine, 33, 561-567.

Lutz, G.E., Palmitier, R.A., \& AN, K.N. (1993) Comparison of tibiofemoral joint forces during open kinetic chain and closed kinetic chain exercises. Journal Bone Joint Surgery, 75, 732-739.

Mafi, N., Lorentzon, R., \& Alfredson, H. (2001). Superior short-term results with eccentric calf muscle training compared to concentric training in a randomized prospective multicenter study on patients with chronic achilles tendinosis. Knee Surgery Sports Traumatology Arthroscopy, 9, 42-47.

Middleton, P., \& Montero, C. (2004). Le travail musculaire excentrique : intérêts dans la prise en charge thérapeutique du sportif. Annales de Réadaptation et Médecine Physique, 47, 282-289.

Middleton, P., Duprey, E., Boudine, M., \& Delest M. (2005). Comment le muscle cicatrise, quel est l'impact de la rééducation? In Hérisson C., Rodineau J. (Eds.), Muscle traumatique et mécanique (p. 145-150). Masson, Paris.

Middleton, P., Moreau, V., Petit, H., \& Montero C. (2010). Récupération musculaire après ligamentoplastie. Facteurs à prendre en compte. Conduite du renforcement musculaire. Lettre Médecine Physique Readaptation, 26, 9-13.

Middleton, P., Gaujard, E., Petit, H., Guillermo, A., Vidal, M.C., Bientz, I., \& Monguillot, P. (2013). Isocinétisme : le travail musculaire excentrique. Lettre Médecine Physique Réadaptation, 29 (2), 70-78.

Mikkelsen, C., Werner, S., \& Eriksson, E. (2000). Closed kinetic chain alone compared to combined open and closed kinetic chain exercises for quadriceps strenghtening after anterior cruciate ligament reconstruction. Knee Surgery Sports Traumatology Arthroscopy, 8 (6), 337-342.

Mouraux, D., \& Brassinne, E. (2009). Place des contractions musculaires excentriques dans le traitement des tendinopathies achilléennes et rotuliennes. In Croisier J.L., Codine P. (Eds.), Exercice musculaire excentrique (p. 131-139). Masson, Paris.

Mouraux, D., \& Brassinne, E. (2011). Intérêt du renforcement musculaire excentrique dans le traitement des tendinopathies. Revue Médicale Bruxelles, 32, 375-380.

Noé, N., Billuart, F., Messina, M., \& Nephtali, J.L. (2010). Effets du travail musculaire sur le phénomène de ligamentisation. Kinésithérapie Revue, 100, 30-34.

Paulos, L., Wnorowski, D., \& Beck, C. (1991). Rehabilitation following knee surgery. Sports Medecine, 11 (4), 257-275.

Puig, P.L., Trouve, P., Savalli, L., Laboute, E., \& Aucagne, C. (2005). La rééducation des ligamentoplasties du LCA au DIDT. Journal Traumatologie Sport, 22, 148-153.

Proske, U., \& Morgan, D.L. (2001). Muscle damage from eccentric exercise: mechanism, mechanical signs, adaptation 
and clinical applications. Journal Physiology, 537 (2), 333-345.

Queiros Da Silva, C. (2004). Apport du travail isocinétique excentrique dans le traitement des lésions musculotendineuses subaiguës. Thèse pour l'obtention du grade de docteur en médecine. Université Claude-Bernard, Lyon 1.

Queiros Da Silva, C., Cotte, T., Vicard, L., Chantelot, L., \& Ferret, J.M. (2005). Apport du travail excentrique isocinétique dans le traitement des tendinopathies calcanéennes et des lésions musculaires de la cuisse. Journal Traumatologie Sport, 22, 219-225.

Rees, J.D., Wolman, R.L., \& Wilson, A. (2009). Eccentric exercises: why do they work, what are the problems and how can we improve them? British Journal Sports Medecine, 43, 242-246.

Stanish, W.D., Rubinovich, R.M., \& Curwin, S. (1986). Eccentric exercise in chronic tendonitis. Clinical Orthopaedics and Releated Research, 208, 65-68.
Thorborg, K. (2012). Why hamstring eccentrics are hamstring essentials. British Journal Sports Medecine, 46, 463-465.

Toutoungi, D.E., Lu, T.W., Leardini, A., Catani, F., \& O'Connor, J.J. (2000). Cruciate ligament forces in the human knee during rehabilitation exercises. Clinical Biomechanics, 15, 176-187.

Walden, M., Hägglund, M., Magnusson, H., \& Ekstrand, J. (2011). Anterior cruciate ligament injury in elite football: a prospective three-cohort study. Knee Surgery Sports Traumatology Arthroscopy, 19, 11-19.

Williams, P.E., \& Goldspink, G. (1971). Longitudinal growth of striated muscle fibers. Journal Cellular Science, 9 (3), 751-767.

Woods, C., Hawkins, R., Hulse, M., Hodson, A., Andersen, T., \& Bahr, R. (2002). The Football association Medical research Programme: an audit of injuries in professionalanalysis of preseason injuries. British Journal Sports Medecine, 36 (6), 436-441. 